\title{
Una evaluación de la esterilización por laparoscopia consecutiva al aborto incompleto.
}

Dr. René Guzmán Serani (1)

Srta. Rhona D. Kantor, M.P.H. (2)

Dr. Leonardo Mandujano (1)

Dr. Carlos Martínez (1)

Srta Norma Haussmann 
la esterilización de todas las mujeres pertenecientes a este estudio e igualmente, para todas ellas la oclusión tubaria se hizo mediante electrocoagulación y sección de ambas trompas.

\section{Procedimiento en el aborto}

La mayoría de los abortos fueron inducidos ilegalmente fuera del hospital mediante la introducción de una sonda de goma en el útero y es así como al momento de su ingreso, solamente el $9,1 \%$ de las pacientes fueron catalogadas como portadoras de un aborto espontáneo.

En el $6.1 \%$ de los casos el aborto fue consirerado séptico y las pacientes, la mayoría de las veces con fiebre superior a $\operatorname{los} 38^{\circ} \mathrm{C}$, fueron sometidas a tratamiento antibiótico previo al raspado. La esterilización fue planeada en estos casos para cuando estuvieran afebriles y en los casos no sépticos ella fue concurrente con o dentro de las primeras 24 horas del raspado uterino.

Procedimiento de la esterilización

Una hora antes de la laparoscopia las pacien- tes fueron preparadas mediante un enema rectal, limpieza abdominal, rasurado y la administración de un sedante, clorpromazina 25 mgrs. intramuscular.

En el pabellón de operaciones se administraron 100 mgrs. de meperidina y 10 mgrs. de diazepam endovenosos. La zona subumbilical fue anestesiada con procaina al $2 \%$ en infiltración local y en algunos casos fue necesario complementar esta anestesia con un barbitúrico endovenoso de acción corta.

Para conseguir pneumoperitoneo se utilizó anhídrido carbónico que fue insuflado a través de un trocar de Thouhy insertado cuatro cms. más abajo del ombligo, a una velocidad de $1.000 \mathrm{ml} / \mathrm{minuto}$ con una presión - máxima de $30 \mathrm{~mm} \mathrm{Hg}$. Durante el procedimiento operatorio se mantuvo un flujo constante de CO2 a una velocidad de $400 \mathrm{ml} / \mathrm{mi}^{-}$ nuto y con un promedio de presión máxima de $10 \mathrm{~mm} \mathrm{Hg}$.

El laparoscopio fue introducido a través de una pequeña incisión subumbilical y las trompas tomadas con una pinza especial y electrocoaguladas en su tercio proximal. Un segmento de aproximadamente $10 \mathrm{~mm}$ fué

\section{T A B L A I}

\section{DISTRIBUCION POR EDAD Y PARIDAD}

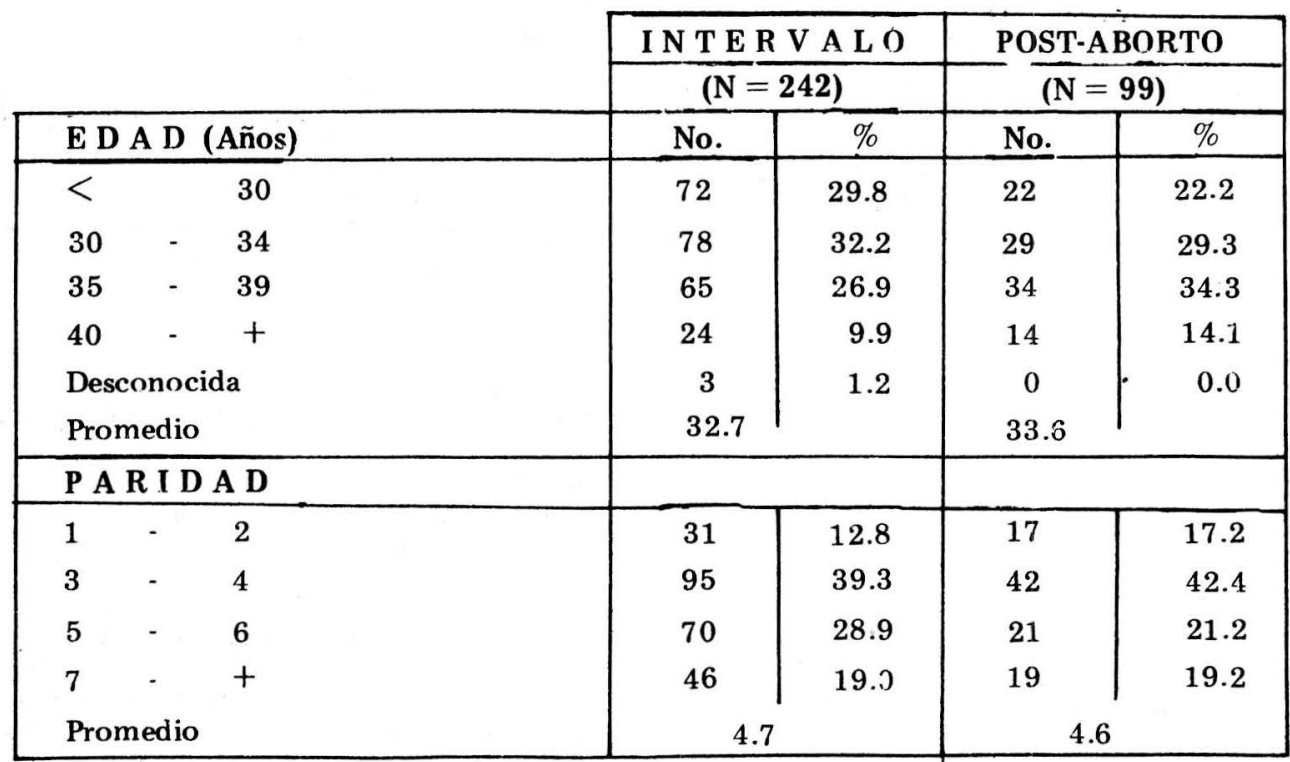


escindido en ambas trompas y los dos cabos restantes fueron recoagulados. La sutura de la incisión abdominal se hizo con dos o tres puntos de catgut simple.

Una vez finalizada la intervención, las pacientes fueron llevadas a una sala de reposo en donde descansaron por lo menos durante cuatro horas. Cuando fue necesario, se administraron analgésicos y aquellas mujeres con mejor stándard socio-económico fueron enviadas a sus casas luego de 6 a 8 horas de reposo. Las mujeres con bajo nivel socio-económico se mantuvieron hospitalizadas por 24 o más horas con el objeto de asegurarles un reposo que probablemente no lograrían en sus domicilios. Todas las pacientes recibieron al ser dadas de alta las indicaciones correspondientes para su cuidado y una citación a control dentro de los próximos 7 a 21 días.

\section{Características de las pacientes}

La edad y paridad de las pacientes fue similar en ambos grupos (Tabla I). El $55.8 \%$ del grupo de esterilizadas en intervalo nunca habían tenido un aborto mientras que todas las pacientes del grupo post-aborto ha- bían tenido por lo menos un aborto antes de la admisión actual. El promedio de abortos de este segundo grupo fue de 2.7. El promedio de hijos vivos fue de 4.0 para el grupo de intervalo y de 4.3 para el grupo esterilizado post aborto. Todas las mujeres tenían por lo menos un hijo vivo.

\section{RESULTADOS}

De fallas técnicas se definen aquellos casos en que la esterilización planeada no pudo ser realizada y ello ocurrió en dos casos del grupo de intervalo en las cuales hubo que realizar una laparotomía. En uno de estos casos el trocar de Thouhy no pudo ser introducido en la cavidad peritoneal y en el otro caso la obesidad de la paciente determinó la imposibilidad de alcanzar las trompas. Estos dos casos han sido excluídos del análisis posterior, ya que requirieron cambio de procedimiento.

Dificultades en el procedimiento pero que no requirieron cambio de él se presentaron. en 23 pacientes $(9.6 \%)$ del grupo de intervalo y en 9 pacientes $(9.1 \%)$ del grupo post aborto (Tabla II).

En lo que se refiere al porcentaje de com-

TABLA II

DIFICULTADES OPERATORIAS

\begin{tabular}{|c|c|c|c|c|}
\hline & \multicolumn{2}{|c|}{ Intervalo } & \multicolumn{2}{|c|}{ Post-aborto } \\
\hline & \multicolumn{2}{|c|}{$\mathrm{N}=240)$} & \multicolumn{2}{|c|}{$(\mathrm{N}=99)$} \\
\hline & No. & $\%$ & No. & $\%$ \\
\hline Adherencias & 6 & 2.5 & 0 & 0.0 \\
\hline Obesidad & 3 & 1.3 & 0 & 0.0 \\
\hline \multicolumn{5}{|l|}{ Otras: } \\
\hline Entrada al peritoneo & 1 & 0.4 & 1 & 1.0 \\
\hline Visualización y/o toma & & & & \\
\hline de trompas & 9 & 3.8 & 7 & 7.1 \\
\hline Oclusión de las trompas & 4 & 1.7 & 1 & 1.0 \\
\hline $\begin{array}{l}\text { Total de casos con dificultades } \\
\text { operatorias }\end{array}$ & 23 & 9.6 & $9^{\bullet}$ & 9.1 \\
\hline
\end{tabular}




\section{T A B L A I I I}

\section{COMPLICACIONES QUIRURGICAS}

\begin{tabular}{|c|c|c|c|c|}
\hline \multirow[b]{3}{*}{ Complicaciones operatorias } & \multicolumn{2}{|c|}{ Intérvalo } & \multicolumn{2}{|c|}{ Post-aborto } \\
\hline & \multicolumn{2}{|c|}{$(\mathrm{N}=240)$} & \multicolumn{2}{|c|}{$(\mathrm{N}=99)$} \\
\hline & No. & $\%$ & No. & $\%$ \\
\hline Sangramiento tubario & 25 & 10.4 & 4 & 4.0 \\
\hline 'Transfusión / hemorragia & 1 & 0.4 & 1 & 1.0 \\
\hline Coagulación de intestino & 0 & 0.0 & 1 & 1.0 \\
\hline Coagulación de vejiga & 2 & 0.8 & 0 & 0.0 \\
\hline Perforación uterina & 1 & 0.4 & 1 & 1.0 \\
\hline Otras* & 5 & 2.1 & 0 & 0.0 \\
\hline $\begin{array}{l}\text { Mujeres con una o más complica- } \\
\text { ciones operitorias }\end{array}$ & 34 & 14.2 & 7 & 7.1 \\
\hline
\end{tabular}

* Ver texto (de la tabla III)

plicaciones durante la cirugía fue el doble más alto en el grupo de intervalo (1.4.2\%) que en el grupo post aborto (7.1\%). Como puede apreciarse en la T'abla III la complicación operatoria más frecuente en ambos grupos fue el sangramiento de una o ambas trompas, pero en todos los casos el sangramiento fue controlado mediante la electrocoagulación.

En el grupo post aborto una mujer sufrió probablemente una quemadura de la pared intestinal y otra una pequeña perforación uterina a nivel de un cuerno durante el raspado. Ambas pacientes quedaron hospitalizadas en observación pero en ninguna de ellas fue necesario ningún tratamiento adicional siendo dadas de alta en buenas condiciones.

En el grupo de intervalo, fueron reportados dos casos de quemadura accidental de la ve. jisa pero ninguno de los dos requirió tratamiento adicional. En otro caso perteneciente a este grupo en el que se sospechó una lesión de la pared uterina, la paciente recibió tratamiento antibiótico profiláctico y ocitócicos, siendo mantenida en observación, pero no requirió de mayores tratamientos ya (que no tuvo complicaciones.
Otras complicaciones reportadas para las pacientes esterilizadas en intervalo fueron un caso de peritonitis química, un caso de insuflación de $\mathrm{CO}_{2}$ extraperitoneal, un caso de electrocoagulación del ligamento redondo, y un caso de hematoma superficial con discreto sangramiento de la herida operatoria. Solo tres pacientes, todas del grupo de in tervalo requirieron de una hospitalización adicional de una noche más, debido a complicaciones. Entre ellas está la enferma con una peritonitis química, una con excesivo sangramiento de la trompa y una tercera paciente que a causa de una anemia importante requirió de una transfusión.

En lo que se refiere a complicaciones derivadas de la anestesia, como vómitos, cefáleas, mareos, etc. fueron infrecuentes ya que estuvieron presentes sólo en el $4 \%$ de las pacientes de intervalo y en un $3 \%$ de las pacientes post aborto. En ninguno de estos casos se requirió tratamiento especial.

El promedio de hospitalización fue de 1.2 noches para las pacientes de intervalo y de 2.1 noches para las pacientes post aborto.

En lo referente al post operatorio inmediato, nueve $(3.8 \%)$ mujeres del grupo de intervalo y tres $(3.0 \%)$ del grupo post aborto 
presentaron dolor abdominal de cierta importancia pero no hubo necesidad de tratamiento especial. Un caso de flebitis, un caso de sangramiento vaginal y un síncope durante este período fueron reportados en el grupo de intervalo.

Como ya se dijo, todas las pacientes recibieron indicación de regresar entre los 7 y 21 días de su alta para un primer control. Sin embargo, el $17.9 \%$ de las pacientes de inter-: valo y el $12.1 \%$ de las del grupo post aborto no cumplieron con este control.

En las pacientes que acudieron a su primer control, encontramos 35 casos $(17.8 \%)$ del grupo de intervalo que acusaron por lo menos una complicación en tanto que en el grupo post aborto esto ocurrió con $10 \mathrm{mu}-$ jeres $(11.5 \%)$. Como puede observarse en la Tabla IV, la complicación más frecuente reportada en ambos grupos fue un estado febril que requirió tratamiento antibiótico en ambos grupos de pacientes.
Entre el momento del alta y la fecha del primer control fijada para 7 días tuvimos la necesidad de rehospitalizar a una paciente del grupo intervalo y a cuatro pacientes del grupo post aborto: La paciente del grupo intervalo tenía una anexoparametritis de etiología desconocida y fue necesario tenerla hospitalizada durante 44 días para cumplir todo su tratamiento debido a que era de procedencia rural, muy alejada de nuestro centro hospitalario. En lo que respecta a las cuatro mujeres del grupo post aborto en las que fue necesaria una rehospitalización, una de ellas presentó un absceso tuboovárico que requirió tratamiento durante 26 días; y otra fue readmitida por una noche con el objeto de repetir el legrado uterino debido a la presencia de restos ovulares.

Las otras dos pacientes del grupo post aborto fueron rehospitalizadas por 14 y 19 días respectivamente a consecuencia de una pél-

T A B L A I V

COMPLICACIONES DETECTADAS EN EL

CONTROL PRECOZ (7-21 DIAS)

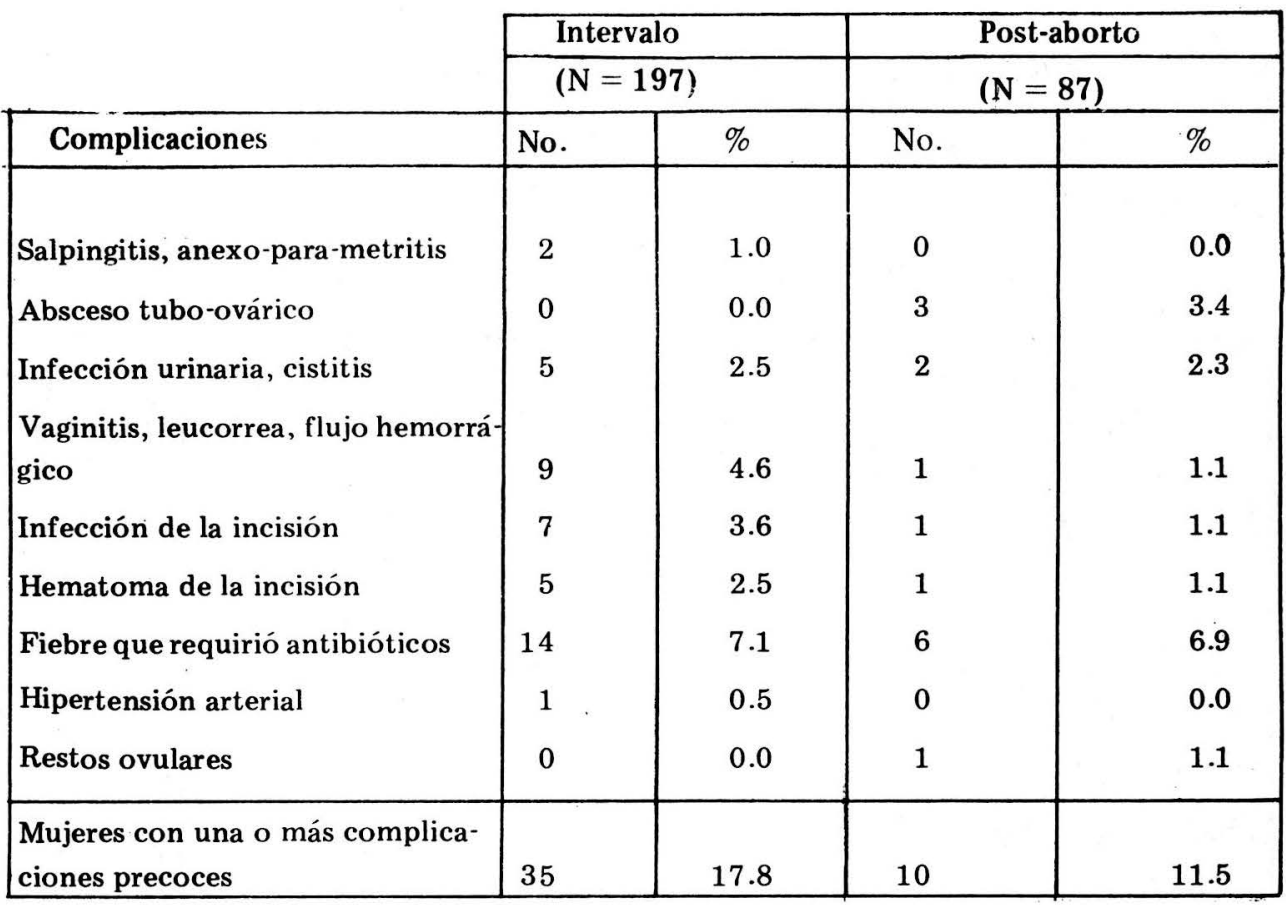


T A B L A V

MOLESTIAS DETECTADAS EN EL

C O N T R O L P R E C OZ $\quad(7-21$ días $)$

\begin{tabular}{|c|c|c|c|c|}
\hline \multirow[b]{3}{*}{ Molestias } & \multicolumn{2}{|c|}{ Intervalo } & \multicolumn{2}{|c|}{ Post-aborto } \\
\hline & \multicolumn{2}{|c|}{$(N=197)$} & \multicolumn{2}{|c|}{$(\mathrm{N}=87)$} \\
\hline & No. & $\%$ & No. & \%o \\
\hline Dolor pelviano o anexial & 8 & 4.1 & 1 & 1.1 \\
\hline Cefalea, dorsalgia & 7 & 3.6 & 0 & 0.0 \\
\hline Gastro-intestinales & 3 & 1.5 & 1 & 1.1 \\
\hline Distensión abdominal & 1 & 0.5 & 0 & 0.0 \\
\hline $\begin{array}{l}\text { Mujeres con una o más moles- } \\
\text { lias }\end{array}$ & 19 & 9.6 & 2 & 2.3 \\
\hline
\end{tabular}

T A B L A V I

C O M P L I C A C I O N E S E N E L C ON TROL A

L OS SEIS MESES

\begin{tabular}{|c|c|c|c|c|}
\hline \multirow[b]{3}{*}{ Complicaciones } & \multicolumn{2}{|c|}{ Intervalo } & \multicolumn{2}{|c|}{ Post-aborto } \\
\hline & \multicolumn{2}{|c|}{$(\mathrm{N}=109)$} & \multicolumn{2}{|c|}{$(\mathrm{N}=74)$} \\
\hline & No. & $\%$ & No. & $\%$ \\
\hline Ca de cuello, displasia & 1 & 0.9 & 2 & 2.7 \\
\hline Cervicitis, endometriosis & 2 & 1.8 & 1 & 1.4 \\
\hline Vaginitis & 4 & 3.7 & 0 & 0.0 \\
\hline Amenorrea & 8 & 7.3 & 2 & 2.7 \\
\hline Metrorragia & 1 & 0.9 & 11 & 0.0 \\
\hline Dolor de cicatriz & 4 & 3.7 & 0 & (). 0 \\
\hline $\begin{array}{l}\text { Mujeres con una ó más } \\
\text { complicaciones }\end{array}$ & 19 & 17.4 & , & $6 . x$ \\
\hline
\end{tabular}


vi-peritonitis. Ambas mujeres tuvieron que ser intervenidas de urgencia y en ambas se encontró un absceso tuboovárico bilateral requiriéndose una anexectomía bilateral y tratamiento antibiótico con penicilina y cloramfenicol en el post operatorio.

El análisis posterior de estos dos casos permitió establecer que ambas mujeres fueron esterilizadas consecutivamente en el mismo pabellón de operaciones y que su complicación no tuvo relación específica ni con el aborto ni con el procedimiento de esterilización siendo muy probablemente el resultado de una infección intrahospitalaria, debido a un equipo incorrectamente esterilizado.

Más de la mitad de las mujeres del grupo de intervalo y el $25.3 \%$ del grupo post aborto no cumplieron con el control programado para los seis meses. Las complicaciones y molestias reportadas por aquellas pacientes de ambos grupos que cumplieron con este control pueden verse en las Tablas VI y VII. El $17.4 \%$ del grupo de intervalo y el $6.8 \%$ del grupo post aborto señalaron por lo menos una complicación en tanto que leves molestias solo fueron reportadas por las pacientes del grupo de intervalo. Las más frecuentes de las molestias referidas fueron cefálea o mareos que se encontraron en el $9.2 \%$ de los casos.
Entre el primero y segundo control, separados por seis meses, no hubo ninguna complicación que requiriera hospitalización de ninguna de las pacientes. Sin embargo, una paciente de cada grupo se embarazó durante este período. En ambos casos el protocolo operatorio había señalado dificultades en visualizar las trompas, en una por obesidad importante y en la otra por adherencias pelvianas. Una de ellas tuvo un aborto inducicido a las trece semanas siendo reesterilizada por la técnica de Pomeroy y la otra paciente tuvo un parto de término, normal.

\section{DISCUSION}

La proporción de dificultades operatorias, complicaciones y morbilidad post-operatoria no fue mayor cuando la esterilización por laparoscopia se combinó con el tratamiento de un aborto inevitable o incompleto. Por el contrario, si observamos el número de complicaciones operatorias en ambos grupos podemos ver claramente que ellas alcanzan a un $14.2 \%$ en el grupo de intervalo y solo a un $7.1 \%$ en el grupo post aborto.

Otro tanto ocurre si comparamos la proporción de complicaciones detectadas en el control precoz a los 7-21 días en el que se puede apreciar un $17.8^{\%}$ de complicaciones para el grupo de intervalo y un $11.5 \%$ para el

\section{T A B L A V I I}

\section{MOLESTIAS EN EL CONTROLA LOS}

\section{SEIS M E S S}

\begin{tabular}{|l|c|c|c|c|}
\cline { 2 - 5 } \multicolumn{1}{c|}{} & \multicolumn{2}{c|}{ Intervalo } & \multicolumn{2}{c|}{ Post-aborto } \\
\cline { 2 - 5 } \multicolumn{1}{c|}{$(\mathrm{N}=109)$} & $\%$ & \multicolumn{2}{c|}{ (N = 74) } \\
\hline Molestias & No. & 1.8 & 0 & 0.0 \\
\hline Dolor anexial & 2 & 9.2 & 0 & 0.0 \\
Cefalea, mareos & 10 & 1.8 & 0 & 0.0 \\
Distensión abdominal & 2 & 1.8 & 0 & 0.0 \\
Colon espásticos & 2 & 13.8 & 0 & 0.0 \\
\hline Mujeres con una ó mas & & 15 & & 0 \\
molestias & & & 0 & 0 \\
\hline
\end{tabular}


grupo post aboito.

Fn lo que se refiere a las complicaciones reportadas en el control de los seis meses, de nuevo nos encontramos con referencias significativas entre ambos grupos: 17, $1 \%$ para el grupo de intervalo y $6.8 \%$ para el grupo post aborto. En ambos grupos de pacientes solo hubo un embarazo y en los dos casos la causa fue una esterilización incompleta debido a mala visualización de las trompas, durante el procedimiento.

En el pasado, ni la inserción de un DIU ni la esterilización tubaria fueron procedimientos reccmendados luego de un aborto séptico o no séptico. Sin embargo, en los últimos años, varios estudios han demostrado que la inserción de un DIU o la esterilización pueden seguir al tratamiento de un aborto in completo sin que por ello aumente el riesgo de complicaciones inmediatas o resultantes de este procedimiento. $(5,7,8)$. Del mismo modo hemos demostrado en el presente estudio que la esterilización por laparoscopia, electrocoagulando y escindiendo ambas trompas, inmediatamente después del tratamiento de un aborto inevitable o incompleto, no parece ser causa de ningún riesgo adicional, ni de complicaciones ni de morbilidad post-operatoria, al compararse con la esterilización en período de intervalo.

El porcentaje de complicaciones para los diferentes tipos de esterilización y de tratamiento del aborto, es variable y altamente dependiente de factores propios de la $\mathrm{pa}^{-}$ ciente, como su estado general, edad, edad del embarazo, etc. Como el aborto es ilegal en Chile, no sabemos cuantos de los abortos de este estudio, iniciados fuera del hospital, fueron provocados o fueron sépticos antes de su hospitalización. Sin embargo, a la luz de los datos de este estudio no nos cabe duda de que el procedimiento combinado de esterilización post-aborto es tan sencillo como el de la esterilización en período de intervalo, igualmente inocuo e igualmente efectivo.

Es por lo tanto necesario no desechar este procedimiento, especialmente en aquellos casos de mujeres tratadas por abortos no sépticos, en las cuales pueden aprovecharse mucho mejor nuestros recursos médicos ya que el procedimiento combinado, es indudablemente ventajoso desde un punto de vista médico y económico.

\section{RESUMEN}

Durante los últimos años, varios estudios han demostrado que la morbilidad y la mortalidad asociados con la esterilización tubaria concurrente con el aborto inducido no son más altas que las encontradas en esterilizaciones efectuadas en ausencia de embarazo. Desde marzo de 1975 hasta mayo de 1977 fueron esterilizadas 341 mujeres por su voluntad mediante laparoscopia, en el Instituto de Obstetricia y Ginecología de la Universidad Austral de Chile. De ellas, 99 $(29.0 \%)$, fueron esterilizadas inmediatamer. te después del legrado uterino realizado como tratamiento de un aborto inevitable o incompleto. Las otras 242 mujeres fueron esterilizadas durante su periodo de intervalo (a lo menos seis semanas después del último parto o aborto). Una perforación simple y única de la pared abdominal con el laparoscopio fue el procedimiento planeado para la esterilización de todas las mujeres pertenecientes a este estudio e igualmente para todas ellas la oclusión tubaria se hizo mediante electrocoagulación y sección de ambas trompas.

Los resuitados mostraron que en dos casos por fallas técnicas no puedieron ser esterilizadas por laparoscopio debido a abesidad en uno, habiendo necesidad de laparotomía. Estos dos casos fueron excluídos del análisis posteriormente por variar procedimiento. El porcentaje de complicaciones fue el doble más alto en el grupo de intervalo $(14.2 \%)$. La complicación más frecuente en ambos grupos sangrado de trompas lo cual fue controlado por electrocoagulación.

\section{SUMMARY}

During the last few years, studies have demonstrated that morbility and mortality associated with induced abortion are not higher than those found in sterilizations carried out when not pregnant. From March 1975 to May 1977341 women were voluntarily sterilized through laparoscopy at the Obstetrics and Ginecology Institute of the Austral University in Chile. Of those, $99(29.0 \%)$ were sterilized inmediately after the scraping of the uterine as treatment for an inevitable or incomplete abortion.t The remaining 242 women were sterilized during an interval (at least 6 weeks after the last birth or abortion). A simple perforation of the abdominal wall with the laparoscopy was the procedure followed for the 
sterilization of all the women of this study, as well as tubal oclusion through electrocuagulation and section of the tubes.

The results showed that in two cases, due to technical failures, they could not be sterilized with laparoscopy due to obesity in one of them, and laparotomy was necessary. These two cases were not included in a later analysis since there was a variation in procedure. The porcentage of complications was twice that of the interval group (14.2\%). The most frecuent complication, in both groups was bleeding of the tubes which was controlled through electrocuagulation.

\section{REFERENCIAS}

1. Amin HK, Neuwirth RS: Further experience with laparoscopic sterilization concomitant, with vacuum curettage for abortion. Fertil Steril 24:592, August 1973.

2. Cheng MCE, Rocnat RW: The safety of combined abortion-sterilization procedure. Am $\mathrm{J}$. Obstet Gynecol 129:548, November 1977.
3. Courey NG, Cunanan RG: Combined laparoscopic sterilization and pregnancy termination. J Reproductive Med 10:291, June 1.973

4. Fishburne JI, Edelman DA, Hulka JF, Mercer JP: Outpatient laparoscopic sterilization with

5. Goldsmith A, Goldberg R, Eyzaguirre H, Lizana $L$.: Inmediate postabortal intrauterine contraceptive device insertion: a doubleblind study. Am J Obstet Gynecol 112:957, 1.972

6. Powe CE, McGee JA: Combined outpatient laparoscopic sterilization with therapeutic abor tion. Am J Obstet Gynecol 126:565, 1.976

7. Quan A, de Badia D, Edelman DA, Goldsmith A: laparoscopic sterilization after "spontaneous" abortion. Int J Gynecol Obstet 15:258, 1.977

8. Quan A, Edelman DA, Goldsmith A, Thomas $M$, Zappala-Badia D: Inmediate post-abortion insertion of the Dalkon Shield. Contraception 12:23, 1.975

9. Whitson LG, Ballard CA, Israel R: Laparoscopic tubal sterilization coincident with therapeutic abortion by suction curettage. Ob Gyn 41:677, May 1.973 